\title{
Emerging human alveolar echinococcosis in Hungary (2003-2018): a retrospective case series analysis from a multi-centre study
}

\author{
Balázs Dezsényi ${ }^{1}$, Zsolt Dubóczki², Tamás Strausz ${ }^{3}$ Eszter Csulak4, Veronika Czoma ${ }^{4}$, Zsolt Káposztás ${ }^{4}$, \\ Mária Fehérvári ${ }^{6}$, Áron Somorácz ${ }^{7}$, András Csilek ${ }^{8}$, Attila Oláh ${ }^{9}$, Kálmán Almási $^{10}$, Attila Patonai ${ }^{11}$, Dénes Görög ${ }^{11}$, \\ Zoltán Széll ${ }^{12}$, Zoltán Tolnai ${ }^{12}$, Tamás Sréter ${ }^{12}$, József Danka ${ }^{13}$, Herbert Auer ${ }^{14}$, Beate Grüner ${ }^{15}$, \\ Thomas F. E. Barth ${ }^{16}$ and Adriano Casulli ${ }^{17,18^{*}}$ (D)
}

\begin{abstract}
Background: Human alveolar echinococcosis (AE) caused by Echinococcus multilocularis is an underreported, often misdiagnosed and mistreated parasitic disease mainly due to its low incidence. The aim of this study was to describe the epidemiological and clinical characteristics of human AE patients in Hungary for the first time.

Method: Between 2003 and 2018, epidemiological and clinical data of suspected AE patients were collected retrospectively from health database management systems.

Results: This case series included a total of 16 AE patients. The mean age of patients was 53 years (range: 24-78 years). The sex ratio was 1:1. Four patients (25\%) revealed no recurrence after radical surgery and adjuvant albendazole (ABZ) therapy. For five patients (31.3\%) with unresectable lesions, a stabilization of lesions with ABZ treatment was achieved. In seven patients (43.8\%), progression of AE was documented. The mean diagnostic delay was 33 months (range: 1-122 months). Three AE related deaths (fatality rate 18.8\%) were recorded.

Conclusions: $A E$ is an emerging infectious disease in Hungary with a high fatality rate since based on our results, almost every fifth AE patient died in the study period. Differential diagnosis and appropriate surgical and medical therapy for AE is an urging challenge for clinicians in Hungary, as well as in some other European countries where E. multilocularis is prevalent.
\end{abstract}

Keywords: Human alveolar echinococcosis, Echinococcus multilocularis, Clinical epidemiology, Case series, Hungary

\section{Background}

Human alveolar echinococcosis $(\mathrm{AE})$ is one of the most dangerous and potentially lethal parasitic zoonosis in the temperate and arctic regions of Europe, which is caused by Echinococcus multilocularis (Em), a small tapeworm belonging to family Taeniidae [1]. Em develops into its adult form

\footnotetext{
* Correspondence: adriano.casulli@iss.it

${ }^{17} \mathrm{WHO}$ Collaborating Centre for the epidemiology, detection and control of cystic and alveolar echinococcosis, Department of Infectious Diseases, Istituto Superiore di Sanità, Rome, Italy

${ }^{18}$ European Union Reference Laboratory for Parasites. Department of Infectious Diseases, Istituto Superiore di Sanità, Rome, Italy

Full list of author information is available at the end of the article
}

in the small intestine of canid definitive hosts, mainly the red fox (Vulpes vulpes) in Europe [2, 3]. To which extent dogs may play a central role in transmitting $\mathrm{AE}$ infection to humans in Europe, is still under debate [4, 5]. Infective Em eggs are mainly released into the environment with the feces of infected foxes and ingested by intermediate hosts, mainly small rodents belonging to family Arvicolinae. Oncospheres hatching from eggs penetrate the intestinal mucosa of Arvicolinae and migrate via portal venous and lymphatic circulation into the capillary bed of the main target organ, namely the liver. The oncospheres develop into a larval metacestode, which slowly grows into an infiltrative parasitic tissue.

C C The Author(s). 2021 Open Access This article is licensed under a Creative Commons Attribution 4.0 International License, which permits use, sharing, adaptation, distribution and reproduction in any medium or format, as long as you give appropriate credit to the original author(s) and the source, provide a link to the Creative Commons licence, and indicate if changes were made. The images or other third party material in this article are included in the article's Creative Commons licence, unless indicated otherwise in a credit line to the material. If material is not included in the article's Creative Commons licence and your intended use is not permitted by statutory regulation or exceeds the permitted use, you will need to obtain permission directly from the copyright holder. To view a copy of this licence, visit http://creativecommons.org/licenses/by/4.0/ The Creative Commons Public Domain Dedication waiver (http://creativecommons.org/publicdomain/zero/1.0/) applies to the data made available in this article, unless otherwise stated in a credit line to the data. 
Humans are dead-end intermediate hosts and may become infected, similarly to small rodents, through the ingestion of Em eggs from contaminated environment or matrices. Human $\mathrm{AE}$ is a rare disease associated with nonspecific symptoms and a long incubation period of several years. Therefore, main drivers of Echinococcus spp. transmission pathways (food-borne, water-borne or hand-to-mouth) and the identification of potential risk factors are still under scientific debate [4-6].

Clinical management of human AE patients is challenging, even for experts. In humans, oncospheres established in the liver develop as aggregated vesicles (appearing as microcysts at imaging) and then evolving into a tumorlike parasitic mass with locally invasive growth pattern, mimicking malignancy. Histologically, AE-lesions are characterized by necrosis with intermingled fragments of the laminated layer surrounded by a granulomatous inflammation [3] (Fig. 1c; Fig. 2a). Initially, AE is often asymptomatic, and the time from infection to the development of a typical AE-liver lesion is 5 to 15 years in immunocompetent individuals. Diagnosis of $\mathrm{AE}$ is based on clinical and epidemiological data, imaging techniques, histopathology and/or nucleic acid detection and serology [7]. Imaging techniques has a central role in the differential diagnosis and clinical management of human AE. In particular, the importance of ultrasonography (US) in hepatic AE rely primarily in the opportunity for early diagnosis [8]. For further characterization of lesions and for investigate extension of $\mathrm{AE}$ lesion to adjacent structures and distant metastases, computed tomography $(\mathrm{CT})$ and magnetic resonance imaging (MRI) are useful $[7,9,10]$. During the past decades, several advanced classification systems has been introduced to distinguish types of $\mathrm{AE}$ lesions based on different imaging characteristics adjusted for conventional imaging techniques (US, MRI, CT) $[8$, 11-15]. For interdisciplinary treatment planning, staging based on image findings according the WHO - Informal Working Group on Echinococcosis (WHO-IWGE) PNM classification is recommended [7]. Albendazole (ABZ) is the first drug of choice for medical treatment and is mandatory in all patients [16, 17]. Radical surgery, aiming to completely remove all lesions including satellite (metastatic) lesions followed by a two-year ABZ administration is the standard treatment aiming for cure. The majority of patients are inoperable and need long-term ABZtreatment $[18,19]$. Interventional procedures should be preferred to palliative surgery whenever possible, and radical surgery is the first choice in all cases suitable for total resection of the lesion(s). Rescue liver transplantation is a therapeutic option for $\mathrm{AE}$ patients with inoperable lesions and/or chronic liver failure $[7,20]$.

In this context, our study aims to collect epidemiological and clinical data on all human AE patients diagnosed in Hungary so far by means of retrospective caseseries analysis to present the consequences of parasite spreading in previously AE free European countries and

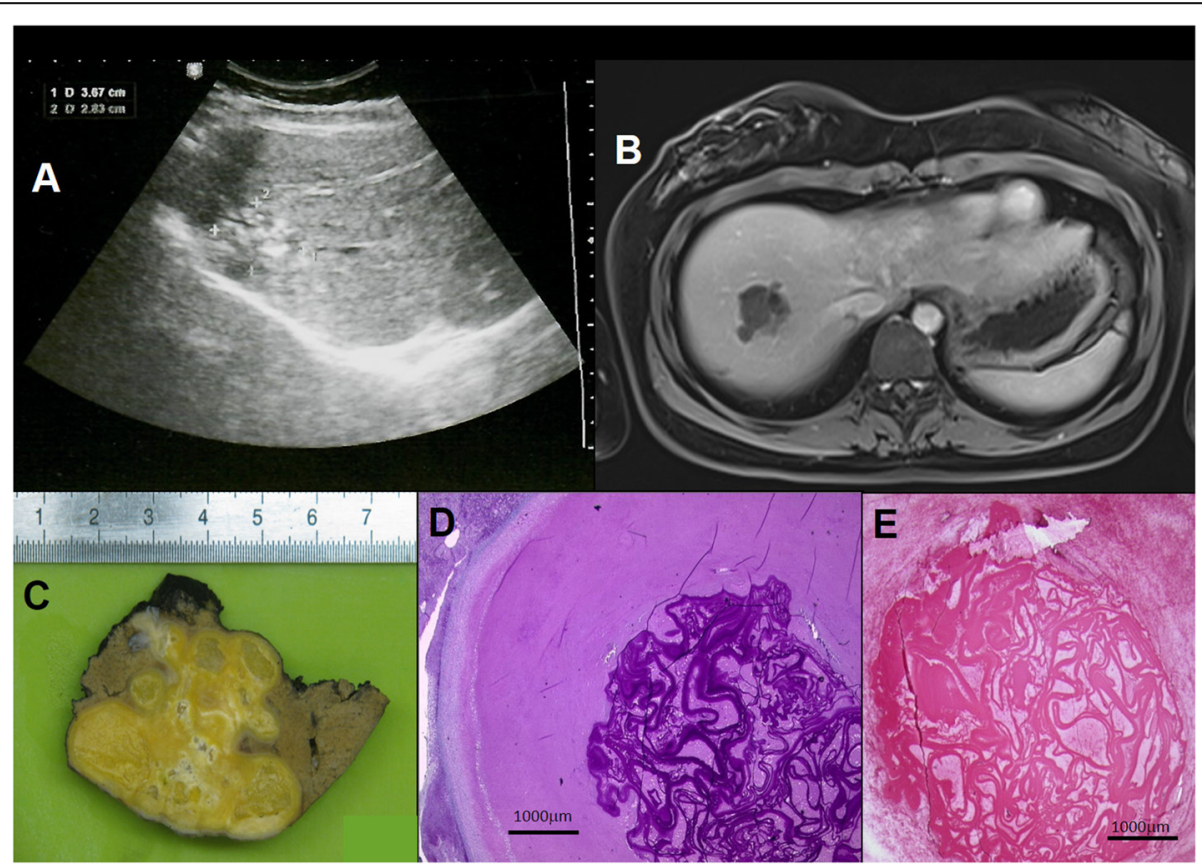

Fig. 1 Patient No. 11. a Ultrasound image of alveolar echinococcosis (AE) lesion in the liver. $\mathbf{b}$ Axial T1 weighted MR image of the same AE lesion. c Gross picture of the lesion removed by segmentectomy. $\mathbf{d}$ Microscopic appearance of the lesion: note the multiplex, slender, PAS positive LL of the metacestode surrounded by abundant necrotic material (PAS). e Immunohistochemical staining using the monoclonal antibody Em2G11: the antibody marks the laminated layer of Em ( $L L$ - red colored band-like structures) 


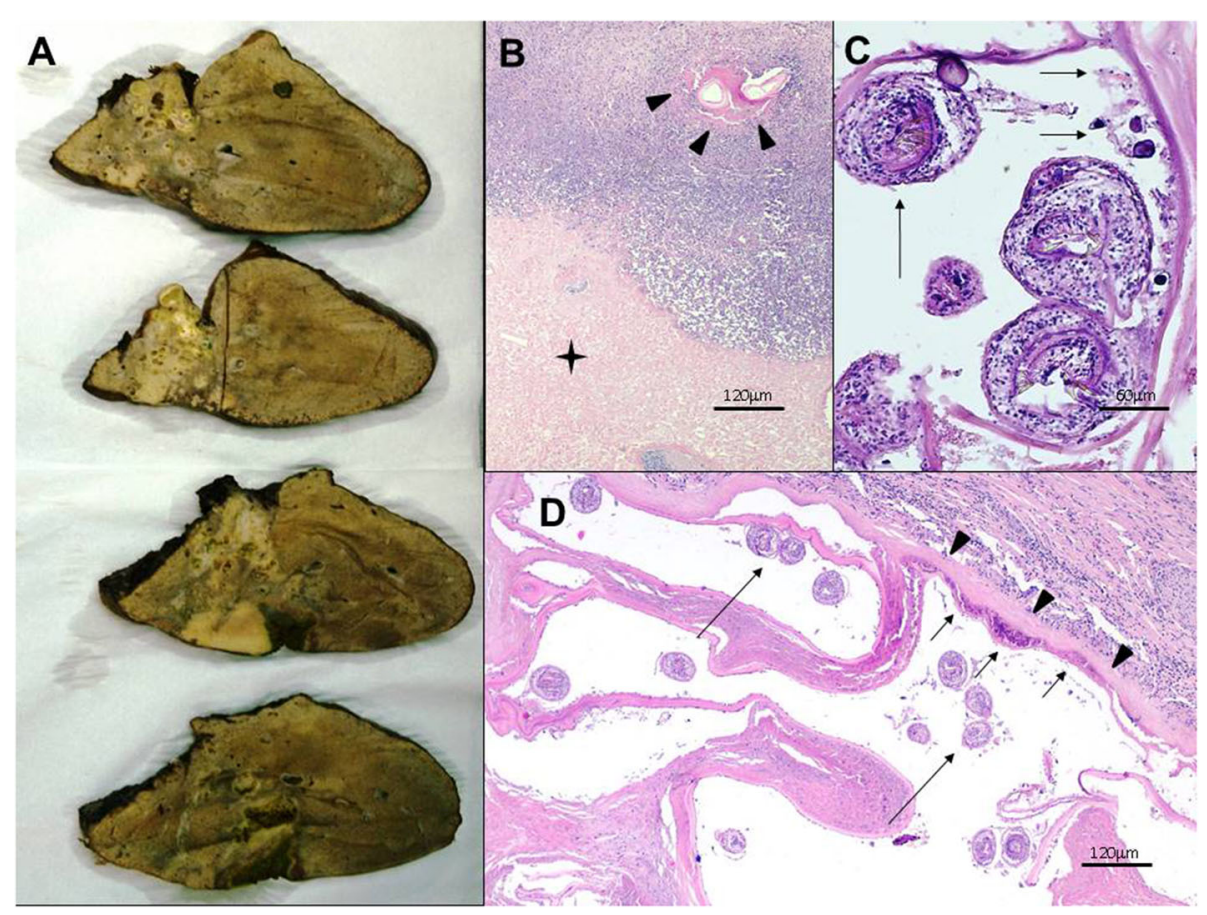

Fig. 2 Patient No. 14. a Gross view of the resected right lobe of the liver shows heterogeneous solid mass lesion that contains externally budding vesicles and calcified foci with a maximum diameter of a single vesicle around $10 \mathrm{~mm}$. b, c, $\mathbf{d}$ Histopathological characteristics of Echinococcus multilocularis lesion in the liver. Normal appearance of the liver is distorted. Severe inflammation is present around the necrotic liver tissue (asterisk in $\mathbf{b}$ ). This inflammatory infiltrate consists of lymphocytes and histiocytes. In some areas fragment of cuticular membrane is observed within the necrotic area (arrowheads in $\mathbf{b}$ ). This membrane displays the laminated layer as a tender band-like structure (arrowheads in $\mathbf{d}$ ) with a germinal layer (short arrows in $\mathbf{c}$ and $\mathbf{d}$ ). Note the invaginated protoscoleces found within the vesicles (long arrows in $\mathbf{c}$ and $\mathbf{d}$ )

provide guidance to clinicians for the management of this emerging helminthic infection in the future.

\section{Methods}

\section{Case selection and inclusion criteria}

A multicentre retrospective case-series analysis was conducted during the study period from $01 / 01 / 2003$ to $31 / 12 / 2018$, describing the epidemiological and clinical characteristics of all patients with probable or confirmed diagnosis of $\mathrm{AE}$ in Hungary.

The Hungarian patients included in this study were those who had positive serology for Em with one highly sensitive (ELISA or IHA) and one highly specific (Western blot) test and therefore fulfilled the clinical and laboratory diagnostic criteria for probable or confirmed AE patient as proposed by the WHO-IWGE [7]. A probable case was defined as any patient with clinical and epidemiological history, and imaging findings, and positive serology for $\mathrm{AE}$ with two positive tests. A confirmed case was defined as the above, plus i) histopathology compatible with $\mathrm{AE}$ and/ or ii) detection of Em nucleic acid sequence(s) in a clinical specimen. Patient(s) who were serologically negative but Em infection was unequivocally confirmed by histopathological methods were also included in this case series.

\section{Epidemiological and serological data}

Baseline demographic and clinical data were collected from medical records from the database management systems of the following centres: National Public Health Centre, National Reference Laboratory for Human Parasitic Diseases, Budapest, Hungary; Central Hospital of Southern Pest National Institute of Hematology and Infectious Diseases, Budapest, Hungary (MedSol); Semmelweis University Department of Transplantation and Surgery, Budapest, Hungary (Medsol); National Institute of Oncology, Budapest, Hungary (MedWorks); Kaposi Mór Teaching Hospital, Kaposvár, Hungary (eMedsol); BorsodAbaúj-Zemplén County Teaching Hospital, Miskolc, Hungary (Medworks); Petz Aladár County Teaching Hospital, Győr, Hungary (Hospitaly) and Jósa András County Teaching Hospital, Nyíregyháza, Hungary (Helise).

Data regarding potential risk factors were collected by means of questionnaires with dichotomous questions delivered to patients providing informed consent. The following potential risk factors on human infection with Em were investigated: dog ownership, playing with dogs, have a kitchen garden, farming occupation, did hay making in meadows not adjacent to water, went to forests for vocational reasons, ate unwashed strawberries, chewed grass, hunting, handling 
foxes, consumption of wild vegetables and fruit [4], and presence of foxes at the place of residence.

Serological data were obtained from the Hungarian Reference Laboratory for Human Parasitic Diseases (National Public Health Centre, Budapest, Hungary). Antibody titers were first determined by a high-sensitivity ELISA test (Hydatidosis ELISA, Vircell, Spain; Ridascreen Echinococcus IgG, R-Biopharm, Germany) or indirect hemagglutination test (Cellognost-Echinococcosis, Siemens, Germany). As a second high-specificity confirmatory test, Western blot (Echinococcus Western blot IgG, Ldbio, France) was applied [21].

\section{Histopathology, immunohistochemistry, and molecular analysis}

Tissue sections from samples included core-biopsies, surgical specimens and specimens from autopsies. Both haematoxylin-eosin (H\&E) and Periodic Acid Schiff (PAS) staining were performed. Histological criteria for diagnosis included the identification of multiple cysts, a PAS positive slender $(<1 \mathrm{~mm})$ laminated layer within abundant necrotic areas, a tubular growth pattern accompanied by a granulomatous cell-reaction without any definite fibrous capsule around the lesion [22] (Fig. 1d-e, Fig. 2b-d). Further confirmation derived from an immunohistochemical staining with the monoclonal antibody Em2G11 (Ulm, Germany) [23] (Fig. 1e) or by the detection of Em specific DNA by PCR method (Wien, Austria). Cytological analysis of samples gained by fine-needle aspiration biopsy was also carried out. $\mathrm{Cy}$ tology alone without further confirmatory tests such as immunocytochemistry was regarded as inappropriate method in diagnosing $\mathrm{AE}$ in this case series.

\section{Clinical data and case follow-up}

For detailed clinical analysis, we registered the date (month, year) of the first symptoms and/or findings; the initial symptoms; the first physical and laboratory findings; the initial and final radiomorphological characteristics of $\mathrm{AE}$ lesions investigated by conventional imaging methods (US, CT or MRI) with the largest diameter of the lesions in millimeters; the preliminary diagnosis; extrahepatic localization of the parasite at first diagnosis; the date (month, year) of diagnosis of probable or confirmed $\mathrm{AE}$ (Table 1). The diagnostic delay in each identified patient as the time period between the first symptoms/findings and the recognition of $\mathrm{AE}$ was calculated. Treatment modalities were also analyzed. ABZ was given at a dose of $800 \mathrm{mg}$ per day in two divided doses continuously or intermittently. Duration of ABZ administration were registered in months. In AE patients with resectable parasitic lesion, curative surgical treatment followed the rules of tumor surgery aiming to perform R0 resection without any parasitic residue. Endoscopic and percutaneous interventions (Table 2) and potentially immunosuppressive co-morbidities were also recorded.

Clinical follow-up period was determined as the time interval between the date of $\mathrm{AE}$ diagnosis and the date of $\mathrm{AE}$ related or unrelated death or the endpoint of this study (31/12/2018). In given cases, the WHO-IWGE PNM classification [7] for AE was carried out based on the initial and final imaging during the study period. We registered $\mathrm{AE}$ associated complications including both the sequelae of the natural course of the infection and the consequences of pharmacological, surgical and/or endoscopic interventions. With regards to the outcome, course of $\mathrm{AE}$ lesions on conventional imaging was assessed as follows: "recurrence/no recurrence" after radical resection regardless of the duration of adjuvant ABZ treatment, "regression" (decreased size or stable size and declining symptoms or disappearance of former complications such as cholestasis, cholangitis), "progression" (increase in size and/or extension to neighboring organs and/or metastases or new clinical complications due to the $\mathrm{AE}$ as cholestasis, need for ERCPintervention, cholangitis), "stabilization" (no change in size of the lesions and no new complications such as cholestasis and cholangitis) [19]. AE related death or AE unrelated death was also used as clinical outcome categories. At the end of the study, assessment of AE lesions was done by conventional imaging and is given as PNM re-staging (Table 1; Table 2).

\section{Results}

\section{Epidemiological features}

Between 2003 and 2018, a total of 16 patients of AE were reported to the National Public Health Centre in Hungary. Based on clinical and laboratory diagnostic criteria (WHO-IWGE expert consensus), 10 were diagnosed as confirmed (62.5\%) and six as probable (37.5\%) AE patients. The sex ratio was 1:1. The mean age of patients at the time of first symptoms or findings related to $\mathrm{AE}$ was 53 years (range: $24-78$ years). Patients originated from rural areas $(n=7 ; 43.8 \%)$, suburban areas $(n=4 ; 25 \%)$, and urban areas $(n=5 ; 31.3 \%)$. Data regarding potential risk factors were collected from 12 out of $16 \mathrm{AE}$ patients. The following potential risk factors were recorded in our series: have a kitchen garden (91.7\%), went to forests for vocational reasons (83.3\%), dog ownership (66.7\%), playing with dogs $(66.7 \%)$, consumption of wild vegetables and fruits $(66.7 \%)$, recognizing foxes at the place of residence (58.3\%), farming occupation (50\%), eat unwashed strawberries (50\%), did haymaking in meadows not adjacent to water $(16.7 \%)$, and chewed grass (16.7\%).

\section{Diagnostic features}

Five patients (31.3\%) were asymptomatic at first examination, and $\mathrm{AE}$ lesions were incidental imaging findings. 
Table 1 Diagnostic features of human alveolar echinococcosis cohort patients in Hungary (2003-2018)

\begin{tabular}{|c|c|c|c|c|}
\hline case no. & 1 & 2 & 3 & 4 \\
\hline $\begin{array}{l}\text { onset of } \\
\text { symptoms or first } \\
\text { findings }\end{array}$ & 09.2001 & $\begin{array}{l}09.2003 \text { focal hepatic lesions } \\
\text { during imaging studies }\end{array}$ & 10.2004 & 08.2008 \\
\hline $\begin{array}{l}\text { initial symptoms } \\
\text { and physical } \\
\text { findings }\end{array}$ & epigastric pain, vomitus & asymptomatic, hepatomegaly & $\begin{array}{l}\text { epigastric and } \\
\text { right } \\
\text { hypochondriac } \\
\text { pain }\end{array}$ & $\begin{array}{l}\text { jaundice, pruritus, right } \\
\text { hypochondriac pain }\end{array}$ \\
\hline $\begin{array}{l}\text { liver function } \\
\text { tests: } \\
\text { liver enzymes (U/ } \\
\text { l); sebi }(\mu \mathrm{mol} / \mathrm{l})\end{array}$ & normal & - & $\begin{array}{l}\text { elevated GGT } \\
\text { (104) }\end{array}$ & $\begin{array}{l}\text { elevated ALP (1254), GGT (570) and } \\
\text { sebi (202) }\end{array}$ \\
\hline $\begin{array}{l}\text { initial US/CT/MRI } \\
\text { (date) } \\
\text { radiomorphology } \\
\text { largest diameter } \\
\text { of } \mathrm{AE} \\
\text { lesion(s) in mm }\end{array}$ & $\begin{array}{l}\text { US }(09.2001)-15 \text { mm hyperreflective } \\
\text { area in SIV CT (03.2003) -echinococcal } \\
\text { cysts inboth lobes, number, size, } \\
\text { localization unknown }\end{array}$ & $\begin{array}{l}\text { US (04.2005) and CT } \\
\text { (08.2009) - } 10 \text { typical } \\
\text { AE lesions in SIV, SV, } \\
\text { SVI, SVIII, 10-30 mm, } \\
\text { largest lesion } 50 \mathrm{~mm}\end{array}$ & $\begin{array}{l}\text { US (10.2004), CT } \\
\text { (11.2004) and MRI } \\
\text { (06.2005) - one } \\
\text { typical } \\
\text { AE lesion - } 100 \\
\text { mm -in SV, SVI, } \\
\text { SVII, SVIII }\end{array}$ & $\begin{array}{l}\text { US (08.2008) - typical central AE } \\
\text { lesion - } 110 \mathrm{~mm} \text { - in the } \\
\text { dichotomy of hepatic common } \\
\text { duct, SIV, SV }\end{array}$ \\
\hline $\begin{array}{l}\text { preliminary } \\
\text { diagnosis }\end{array}$ & echinococcosis & $\begin{array}{l}\text { liver tumor, } \\
\text { echinococcosis }\end{array}$ & $\begin{array}{l}\text { liver tumor, } \mathrm{HCC} \text {, } \\
\text { liver metastasis }\end{array}$ & $\begin{array}{l}\text { liver tumor, } \\
\text { adenocarcinoma }\end{array}$ \\
\hline $\begin{array}{l}\text { serology } \\
\text { Westernblot } \\
\text { (Ldbio) P3 Em }\end{array}$ & positive & positive & positive & positive \\
\hline $\begin{array}{l}\text { core biopsy/ } \\
\text { surgical sample/ } \\
\text { autopsy }\end{array}$ & - & - & $\begin{array}{l}\text { core biopsy }(2 x) \\
\text { surgical sample } \\
(1 x)\end{array}$ & $\begin{array}{l}\text { core biopsy during } \\
\text { PTC, surgical sample }\end{array}$ \\
\hline $\begin{array}{l}\text { histopathology/ } \\
\text { IH/PCR }\end{array}$ & - & - & $\begin{array}{l}\text { histopathology } \\
\text { and PCR }\end{array}$ & $\begin{array}{l}\text { histopathology } \\
\text { and PCR }\end{array}$ \\
\hline type of diagnosis & probable & probable & confirmed & confirmed \\
\hline $\begin{array}{l}\text { month.year of } \\
\text { diagnosis }\end{array}$ & 04.2003 & 04.2004 & 07.2005 & 09.2008 \\
\hline $\begin{array}{l}\text { latency of } \\
\text { diagnosis (in } \\
\text { months) }\end{array}$ & 20 & 8 & 10 & 2 \\
\hline $\begin{array}{l}\text { extrahepatic } \\
\text { localizationat the } \\
\text { time of diagnosis }\end{array}$ & no pulmonary lesion & no pulmonary lesion & $\begin{array}{l}\text { peritoneal } \\
\text { dissemination } \\
\text { no pulmonary } \\
\text { lesion }\end{array}$ & no pulmonary lesion \\
\hline PNM at diagnosis & PxNxMx & P1NOMx & P2N1Mx & P3N0Mx \\
\hline case no. & 5 & 6 & 7 & 8 \\
\hline $\begin{array}{l}\text { onset of } \\
\text { symptoms orfirst } \\
\text { findings }\end{array}$ & $\begin{array}{l}2002 \text { asymptomatic } \\
\text { hepatic cyst; patient } \\
\text { denied investigations }\end{array}$ & 11.2011 & 12.2012 & 11.2012 \\
\hline $\begin{array}{l}\text { initial symptoms } \\
\text { andphysical } \\
\text { findings }\end{array}$ & $\begin{array}{l}\text { right hypochondriac } \\
\text { pain, vomitus, anasarca, } \\
\text { palpable liver tumor } \\
(12.2010)\end{array}$ & $\begin{array}{l}\text { right hypochondriac } \\
\text { pain, weightloss, } \\
\text { hepatomegaly }\end{array}$ & $\begin{array}{l}\text { asymptomatic, } \\
\text { mild } \\
\text { hepatomegaly }\end{array}$ & asymptomatic \\
\hline $\begin{array}{l}\text { liver function } \\
\text { tests: } \\
\text { liver enzymes (U/ } \\
\text { l); } \\
\text { sebi }(\mu \mathrm{mol} / \mathrm{l})\end{array}$ & $\begin{array}{l}\text { AST (177), ALT (177), } \\
\text { GGT (920), ALP (1152), } \\
\text { sebi (16) }\end{array}$ & $\begin{array}{l}\text { GGT (105), ALP (543), } \\
\text { sebi }(7,1)\end{array}$ & $\begin{array}{l}\text { GGT }(335), \text { ALP } \\
(999), \\
\text { sebi }(7,3)\end{array}$ & normal \\
\hline $\begin{array}{l}\text { initial US/CT/MRI } \\
\text { (date) } \\
\text { radiomorphology } \\
\text { largest diameter } \\
\text { of } \mathrm{AE} \\
\text { lesion(s) in mm }\end{array}$ & $\begin{array}{l}\text { US (12.2010) and CT } \\
\text { (01.2011) two } \\
\text { interconnected pseudocystic AE } \\
\text { lesionsin both lobes - } 130 \mathrm{~mm} \text { and } \\
120 \mathrm{~mm} \text { - dilatated intrahepatic } \\
\text { bileducts }\end{array}$ & $\begin{array}{l}\text { US }(11.2011) \text { and CT } \\
\text { (12.2011) typical AE } \\
\text { lesion in SV, SVI }-83 \mathrm{~mm}- \\
\text { and some } \\
\text { smaller lesions }\end{array}$ & $\begin{array}{l}\text { CT (04.2013) } \\
\text { typical } \\
\text { AE lesion in right } \\
\text { lobe, } \\
135 \mathrm{~mm}, \\
\text { periportal biliary } \\
\text { and vascular }\end{array}$ & $\begin{array}{l}\text { CT (11.2012) and MRI } \\
\text { (08.2014) multiplying small calcified } \\
\text { lesions in SV, SVI, SVII, SVIII }\end{array}$ \\
\hline
\end{tabular}


Table 1 Diagnostic features of human alveolar echinococcosis cohort patients in Hungary (2003-2018) (Continued)

\begin{tabular}{|c|c|c|c|c|}
\hline case no. & 1 & 2 & 3 & 4 \\
\hline & & & $\begin{array}{l}\text { involvement } \\
\text { (right v. } \\
\text { portae, v. } \\
\text { hepatica } \\
\text { intermedia) }\end{array}$ & \\
\hline $\begin{array}{l}\text { preliminary } \\
\text { diagnosis }\end{array}$ & metastasis, tumor, CE & hemangioma, tumor, CE & $\begin{array}{l}\text { cholangiocellular } \\
\text { carcinoma }\end{array}$ & liver metastasis \\
\hline $\begin{array}{l}\text { serology } \\
\text { Westernblot } \\
\text { (Ldbio) P3 Em }\end{array}$ & positive & equivocal & positive & negative (postoperatively $2 \mathrm{x}$ ) \\
\hline $\begin{array}{l}\text { core biopsy/ } \\
\text { surgical sample/ } \\
\text { autopsy }\end{array}$ & $\begin{array}{l}\text { parasitology and cytology from lesion } \\
\text { fluid (FNAB) negative }\end{array}$ & corebiopsy & corebiopsy & corebiopsy and surgical sample \\
\hline $\begin{array}{l}\text { histopathology/ } \\
\text { IH/PCR }\end{array}$ & - & histopathology & histopathology & histopathology andIIH \\
\hline type of diagnosis & probable & confirmed & confirmed & confirmed \\
\hline $\begin{array}{l}\text { month.year of } \\
\text { diagnosis }\end{array}$ & 03.2011 & 01.2012 & 04.2013 & 10.2014 \\
\hline $\begin{array}{l}\text { latency of } \\
\text { diagnosis (in } \\
\text { months) }\end{array}$ & 111 & 1 & 5 & 24 \\
\hline $\begin{array}{l}\text { extrahepatic } \\
\text { localizationat the } \\
\text { time of diagnosis }\end{array}$ & no pulmonary lesion & no pulmonary lesion & $\begin{array}{l}\text { undignified } \\
\text { pulmonary } \\
\text { lesions }\end{array}$ & no \\
\hline PNM at diagnosis & P4NOMx & P2NOMx & P4N0Mx & P1NOMO \\
\hline case no. & 9 & 10 & 11 & 12 \\
\hline $\begin{array}{l}\text { onset of } \\
\text { symptoms or first } \\
\text { findings }\end{array}$ & 10.2013 & 04.2012 & 02.2017 & 03.2017 \\
\hline $\begin{array}{l}\text { initial symptoms } \\
\text { and physical } \\
\text { findings }\end{array}$ & right hypochondriac pain, urticaria & $\begin{array}{l}\text { right hypochondriac pain, } \\
\text { hepatomegaly }\end{array}$ & $\begin{array}{l}\text { epigastric pain, } \\
\text { vomitus }\end{array}$ & right hypochondriac pain \\
\hline $\begin{array}{l}\text { liver function } \\
\text { tests: } \\
\text { liver enzymes (U/ } \\
\text { l); } \\
\text { sebi }(\mu \mathrm{mol} / \mathrm{l})\end{array}$ & ALP (125), GGT (86) & normal & normal & elevated ALP \\
\hline $\begin{array}{l}\text { initial US/CT/MRI } \\
\text { (date) } \\
\text { radiomorphology } \\
\text { largest diameter } \\
\text { of AE lesion(s) in } \\
\mathrm{mm}\end{array}$ & $\begin{array}{l}\text { MRI (12.2015) and CT } \\
(01.2016) 2 \text { typical AE } \\
\text { lesions in the dichotomy of hepatic } \\
\text { veins; in SV/SIVB } 55 \mathrm{~mm} \text {; in SVIII/IVA } 53 \\
\mathrm{~mm}\end{array}$ & $\begin{array}{l}\mathrm{CT}(04.2012) \text { and } \mathrm{MRI} \\
\text { (10.2012) typical AE } \\
\text { lesion in SIV } 42 \mathrm{~mm}\end{array}$ & $\begin{array}{l}\text { US (02.2017), CT } \\
(02.2017) \text { and MRI } \\
\text { (03.2017) two AE } \\
\text { lesions in SVIII } 44 \\
\mathrm{~mm} \\
\text { and in SVII } 12 \\
\mathrm{~mm}\end{array}$ & $\begin{array}{l}\text { US (05.2017), } \\
\text { CT (05.2017) multiplex } \\
\text { AE lesions in both } \\
\text { lobes, } 40 \mathrm{~mm}\end{array}$ \\
\hline $\begin{array}{l}\text { preliminary } \\
\text { diagnosis }\end{array}$ & $\begin{array}{l}\text { atypical rare malignancy liver } \\
\text { metastasis }\end{array}$ & $\begin{array}{l}\text { hemangioma, adenoma, liver } \\
\text { tumor }\end{array}$ & $\begin{array}{l}\text { hemangioma } \\
\text { cholangiocellular } \\
\text { carcinoma, } \\
\text { fibrolamellar } \\
\text { carcinoma }\end{array}$ & $\begin{array}{l}\text { liver metastasis, sarcoidosis, } \\
\text { granulomatous hepatitis }\end{array}$ \\
\hline $\begin{array}{l}\text { serology } \\
\text { Westernblot } \\
\text { (Ldbio) P3 Em }\end{array}$ & positive & positive & $\begin{array}{l}\text { Echinococcus } \\
\text { genus P5 }\end{array}$ & positive \\
\hline $\begin{array}{l}\text { core biopsy/ } \\
\text { surgical sample/ } \\
\text { autopsy }\end{array}$ & - & - & $\begin{array}{l}\text { (FNAB) and } \\
\text { surgical sample }\end{array}$ & corebiopsy $(2 x)$ \\
\hline $\begin{array}{l}\text { histopathology/ } \\
\text { IH/PCR }\end{array}$ & - & - & $\mathbb{H}$ & histopathology \\
\hline type of diagnosis & probable & probable & confirmed & confirmed \\
\hline
\end{tabular}


Table 1 Diagnostic features of human alveolar echinococcosis cohort patients in Hungary (2003-2018) (Continued)

\begin{tabular}{|c|c|c|c|c|}
\hline case no. & 1 & 2 & 3 & 4 \\
\hline $\begin{array}{l}\text { month.year of } \\
\text { diagnosis }\end{array}$ & 01.2016 & 06.2016 & 05.2017 & 07.2017 \\
\hline $\begin{array}{l}\text { latency of } \\
\text { diagnosis (in } \\
\text { months) }\end{array}$ & 28 & 50 & 4 & 5 \\
\hline $\begin{array}{l}\text { extrahepatic } \\
\text { localizationat the } \\
\text { time of diagnosis }\end{array}$ & no & no & no & no \\
\hline PNM at diagnosis & P3N0M0 & P1NOMO & P1NOMO & P2NOMO \\
\hline case no. & 13 & 14 & 15 & 16 \\
\hline $\begin{array}{l}\text { onset of } \\
\text { symptoms orfirst } \\
\text { findings }\end{array}$ & 09.2017 & 09.2016 & 04.2008 & 2008 \\
\hline $\begin{array}{l}\text { initial symptoms } \\
\text { and physical } \\
\text { findings }\end{array}$ & $\begin{array}{l}\text { right hypochondriac } \\
\text { pain, hepatomegaly }\end{array}$ & asymptomatic & asymptomatic & $\begin{array}{l}\text { right hypochondriac } \\
\text { pain }\end{array}$ \\
\hline $\begin{array}{l}\text { liver function } \\
\text { tests: } \\
\text { liver enzymes (U/ } \\
\text { l); } \\
\text { sebi }(\mu \mathrm{mol} / \mathrm{l})\end{array}$ & elevated liver enzymes & GGT (115) & - & - \\
\hline $\begin{array}{l}\text { initial US/CT/MRI } \\
\text { (date) } \\
\text { radiomorphology } \\
\text { largest diameter } \\
\text { of AE } \\
\text { lesion(s) in mm }\end{array}$ & $\begin{array}{l}\text { US (10.2017), CT } \\
\text { (10.2017) typical AE } \\
\text { lesion in SV } 80 \mathrm{~mm}\end{array}$ & $\begin{array}{l}\text { MRI (09.2016) } 15 \mathrm{~mm} \\
\text { wide hypodens area in right } \\
\text { lobe, CT (09.2017) and MRI } \\
\text { (11.2017) } 75 \mathrm{~mm} \text { typical AE } \\
\text { lesion in SV and SVIII, } \\
\text { dilatation of intrahepatic } \\
\text { bileducts }\end{array}$ & $\begin{array}{l}\text { US (04.2008), CT } \\
\text { (07.2008) typical } \\
\mathrm{AE} \\
\text { lesion in SV - } 54 \\
\mathrm{~mm} \text {-and three } \\
\text { small calcified } \\
\text { lesions }\end{array}$ & $\begin{array}{l}\text { US (2008) } 20 \mathrm{~mm} \text { hyperechoic liver } \\
\text { lesion, CT (10.2016) and MRI } \\
\text { (06.2017) } 120 \mathrm{~mm} \text { typical AE lesion } \\
\text { in right lobe (SV-VI-VIII) }\end{array}$ \\
\hline $\begin{array}{l}\text { preliminary } \\
\text { diagnosis }\end{array}$ & liver tumor & $\begin{array}{l}\text { cholangiocellular carcinoma, } \\
\text { Klatskin tumor }\end{array}$ & $\begin{array}{l}\text { atypical hepatic } \\
\text { cyst }\end{array}$ & hemangioma, cystadenocarcinoma \\
\hline $\begin{array}{l}\text { serology } \\
\text { Westernblot } \\
\text { (Ldbio) P3 Em }\end{array}$ & positive & positive & positive & positive \\
\hline $\begin{array}{l}\text { core biopsy/ } \\
\text { surgical sample/ } \\
\text { autopsy }\end{array}$ & $-($ FNAB 2x) & surgical sample & $\begin{array}{l}\text { surgical sample } \\
\text { autopsy }\end{array}$ & surgical sample \\
\hline $\begin{array}{l}\text { histopathology/ } \\
\text { IH/PCR }\end{array}$ & - & histopathology & histopathology & histopathology \\
\hline type of diagnosis & probable & confirmed & confirmed & confirmed \\
\hline $\begin{array}{l}\text { month.year of } \\
\text { diagnosis }\end{array}$ & 12.2017 & 01.2018 & 05.2018 & 08.2018 \\
\hline $\begin{array}{l}\text { latency of } \\
\text { diagnosis (in } \\
\text { months) }\end{array}$ & 4 & 17 & 122 & $115(+12)$ \\
\hline $\begin{array}{l}\text { extrahepatic } \\
\text { localizationat the } \\
\text { time of diagnosis }\end{array}$ & no pulmonary lesion & $\begin{array}{l}\text { subphrenic abscess, peribiliar } \\
\text { vascular invasion, no } \\
\text { pulmonary lesion }\end{array}$ & $\begin{array}{l}\text { falciform } \\
\text { ligament, no } \\
\text { pulmonary lesion }\end{array}$ & no pulmonary lesion \\
\hline PNM at diagnosis & P4NOMO & P4N1Mx & P4N1Mx & P4NOMx \\
\hline
\end{tabular}

E.m Echinococcus multilocularis, AE alveolar echinococcosis, CE cystic echinococcosis, $v$ vena, $d$ ductus, AST aspartate aminotransferase, ALT alanine aminotransferase, GGT gamma-glutamyltransferase, ALP alkaline phosphatase, sebi serum bilirubin, US ultrasound, CT computer tomography, MRI magnetic resonance imaging, IH immunohistochemistry using monoclonal antibody mAbEm2G11, $P C R$ polymerase chain reaction, $t x$ treatment, EPI endoscopic and percutaneous interventions, ERCP endoscopic retrograde cholangiopancreatography, PTC percutaneous transhepatic cholangiography, PTD percutaneous transhepatic drainage, $F N A B$ fine needle aspiration biopsy, $S$ liver segment, $A B Z$ albendazole, $H C C$ hepatocellular carcinoma

In symptomatic cases, the most frequent clinical signs were epigastric and/or right hypochondriac pain. Hepatomegaly, vomitus, weight loss and pruritus were observed in six patients (37.5\%), while palpable liver mass was detected in one patient (6.3\%). Further physical signs were urticaria and anasarca. One patient (stage P3N0Mx) had jaundice (sebi $202 \mu \mathrm{mol} / \mathrm{l}$ ) at first presentation (6.3\%). Nine patients $(56.3 \%)$ had elevated liver 
Table 2 Therapeutic features of human alveolar echinococcosis cohort patients in Hungary(2003-2018)

\begin{tabular}{|c|c|c|c|c|}
\hline case no. & 1 & 2 & 3 & 4 \\
\hline $\begin{array}{l}\text { antiparasitic drug tx } \\
\text { (duration in months) }\end{array}$ & - & ABZ (3) 10.2004-12.2004 & $\begin{array}{l}\text { ABZ (162) continuously } \\
\text { since } \\
07.2005\end{array}$ & $\begin{array}{l}\text { ABZ (12) } 11.2008- \\
01.2009 \text { and } 06.2016- \\
03.2017\end{array}$ \\
\hline surgery & - & - & exploration -unresectable & $\begin{array}{l}\text { exploration, } \\
\text { fenestration, marsupialisation }\end{array}$ \\
\hline EPI & - & - & - & PTD $(2 x)$, ERCP \\
\hline $\begin{array}{l}\text { follow-up period in } \\
\text { months }\end{array}$ & 27 & 177 & 162 & 124 \\
\hline $\begin{array}{l}\text { radiomorphology on } \\
\text { final control, largest } \\
\text { diameter of AE } \\
\text { lesion(s) in mm (date) }\end{array}$ & $\begin{array}{l}\mathrm{CT}(04.2005) \text { - pseudocystic AE } \\
\text { lesion in left lobe } 49 \mathrm{~mm} \text {, two } \\
\text { more AE lesions in right lobe, } 35 \\
\mathrm{~mm} \text { and } 24 \mathrm{~mm}\end{array}$ & US (09.2014) stabilization & MRI (12.2018) stabilization & $\begin{array}{l}\text { US (12.2018) residual cavity } \\
\text { in SIV } 70 \mathrm{~mm} \text {, giant biloma } \\
\text { in porta hepatis }\end{array}$ \\
\hline PNM at final imaging & P1N0Mx & P1NxMx & P2N1Mx & P3N0Mx \\
\hline complications & $\begin{array}{l}\text { elevated GGT (136), ALP (621), } \\
\text { sebi }(36,3)\end{array}$ & - & - & $\begin{array}{l}\text { central biliary obstruction, } \\
\text { cholangitis, biloma, bile- } \\
\text { leaking }\end{array}$ \\
\hline outcome & progression, AE unrelated death & stabilization & stabilization & progression \\
\hline case no. & 5 & 6 & 7 & 8 \\
\hline $\begin{array}{l}\text { antiparasitic drug tx } \\
\text { (duration in months) }\end{array}$ & - & ABZ (5) 02.2012-07.2012 & $\begin{array}{l}\text { ABZ (67) continuously } \\
\text { since } 06.2013\end{array}$ & ABZ (24) postoperatively \\
\hline surgery & marsupialization, drainage & $\begin{array}{l}\text { extended right } \\
\text { hemihepatectomy }\end{array}$ & - & segmentectomy \\
\hline EPI & $\begin{array}{l}\text { ERCP - biliary stent implantation, } \\
\text { nasobiliary stent }\end{array}$ & - & - & - \\
\hline $\begin{array}{l}\text { follow-up period in } \\
\text { months }\end{array}$ & 9 & 84 & 69 & 51 \\
\hline $\begin{array}{l}\text { radiomorphology on } \\
\text { final control, largest } \\
\text { diameter of AE } \\
\text { lesion(s) in mm (date) }\end{array}$ & $\begin{array}{l}\text { US (11.2011) residual cavity } 45 \\
\text { mm, atrophy of right lobe }\end{array}$ & US (02.2018) no recurrence & $\begin{array}{l}\text { MRI (10.2018) and US } \\
(10.2018), 109 \mathrm{~mm}\end{array}$ & US (07.2018) no recurrence \\
\hline PNM at final imaging & P4NOMx & PONOMX & P4NOMx & PONOMO \\
\hline complications & $\begin{array}{l}\text { central biliary obstruction, bile- } \\
\text { leaking, bilio-peritoneal fistula, in- } \\
\text { jury of bileducts during surgical } \\
\text { manipulation, cachexia }\end{array}$ & $\begin{array}{l}\text { postoperative peritonitis, } \\
\text { haematoma, bile-leaking, } \\
\text { Kehr-drainage }\end{array}$ & $\begin{array}{l}\text { v. cava inferior } \\
\text { compression }\end{array}$ & - \\
\hline outcome & progression, $\mathrm{AE}$ related death & no recurrence & stabilization & no recurrence \\
\hline case no. & 9 & 10 & 11 & 12 \\
\hline $\begin{array}{l}\text { antiparasitic drug tx } \\
\text { (duration in months) }\end{array}$ & $\begin{array}{l}\text { ABZ (3) lowered dose } \\
\text { intermittently in } 2016 \text {, finally } \\
\text { ceased }\end{array}$ & $\begin{array}{l}\text { ABZ (30) continuously since } \\
07.2016\end{array}$ & ABZ (21) postoperatively & ABZ (3) 09.2017-11.2017 \\
\hline surgery & - & - & segmentectomy & - \\
\hline EPI & - & - & - & - \\
\hline $\begin{array}{l}\text { follow-up period in } \\
\text { months }\end{array}$ & 36 & 30 & 20 & 18 \\
\hline $\begin{array}{l}\text { radiomorphology on } \\
\text { final control, largest } \\
\text { diameter of AE } \\
\text { lesion(s) in mm (date) }\end{array}$ & $\begin{array}{l}\text { MRI (10.2018), CT (10.2018) no } \\
\text { progression in liver, new } \\
\text { pulmonary micronodules } \\
(09.2017)\end{array}$ & $\begin{array}{l}\text { MRI (11.2018) SIV } 70 \mathrm{~mm} \text {, } \\
\text { progression }\end{array}$ & $\begin{array}{l}\text { MRI (06.2018), US } \\
\text { (10.2018) no recurrence }\end{array}$ & $\begin{array}{l}\text { US }(07.2018) \text { AE lesion in left } \\
\text { lobe } 65 \mathrm{~mm} \text {, AE lesion in } \\
\text { right lobe } 44 \mathrm{~mm}\end{array}$ \\
\hline PNM at final imaging & P3N0Mx & P1N0Mx & PONOMX & P2NOMx \\
\hline complications & $\begin{array}{l}\text { ABZ hepatotoxicity and allergic } \\
\text { reactions, undignified pulmonary } \\
\text { microlesions }\end{array}$ & - & - & - \\
\hline outcome & stabilization & progression & no recurrence & progression \\
\hline case no. & 13 & 14 & 15 & 16 \\
\hline
\end{tabular}


Table 2 Therapeutic features of human alveolar echinococcosis cohort patients in Hungary(2003-2018) (Continued)

\begin{tabular}{|c|c|c|c|c|}
\hline case no. & 1 & 2 & 3 & 4 \\
\hline $\begin{array}{l}\text { antiparasitic drug tx } \\
\text { (duration in months) }\end{array}$ & $\begin{array}{l}\text { ABZ (12) continuously since } \\
01.2018\end{array}$ & $\begin{array}{l}\text { ABZ (12) continuously since } \\
01.2018\end{array}$ & ABZ (3) 06.2018-09.2018 & - \\
\hline surgery & - & $\begin{array}{l}\text { right hemihepatectomy, } \\
\text { exstirpation of d. } \\
\text { choledochus and } \\
\text { cholecystectomy, } \\
\text { hepaticojejunostomia }\end{array}$ & explorative laparotomy & $\begin{array}{l}\text { right hemihepatectomy, } \\
\text { exstirpation of d. } \\
\text { choledochus and } \\
\text { cholecystectomy, } \\
\text { hepaticojejunostomia }\end{array}$ \\
\hline EPI & - & ERCP & $\begin{array}{l}\text { ERCP }(2 x) \text {, stent } \\
\text { implantation }(2 x)\end{array}$ & $\begin{array}{l}\text { ERCP, stent implantation, } \\
\text { PTD }(2 x)\end{array}$ \\
\hline $\begin{array}{l}\text { follow-up period in } \\
\text { months }\end{array}$ & 13 & 12 & 5 & 1 \\
\hline $\begin{array}{l}\text { radiomorphology on } \\
\text { final control, largest } \\
\text { diameter of } \mathrm{AE} \\
\text { lesion(s) in } \mathrm{mm} \text { (date) }\end{array}$ & $\begin{array}{l}\text { MRI (10.2018) AE lesion in SV } 79 \\
\text { mm }\end{array}$ & CT (10.2018) no recurrence & $\begin{array}{l}\text { US (05.2018) } 120 \text { mm AE } \\
\text { lesion occupying left lobe, } \\
\text { ascites, dilatated } \\
\text { intrahepatic bileducts }\end{array}$ & - \\
\hline PNM at final imaging & P4NOMx & P0N1Mx & P4N1Mx & PONOMX \\
\hline complications & $\begin{array}{l}\text { thrombosis and parasitic } \\
\text { infiltration of right v. portae, } \\
\text { compression of } d \text {. hepaticus } \\
\text { dexter }\end{array}$ & $\begin{array}{l}\text { leukopenia, hairloss, } \\
\text { haematoma in residual left } \\
\text { lobe ( } 32 \mathrm{~mm}) \text { and } \\
\text { undignified pulmonary } \\
\text { microlesions }\end{array}$ & $\begin{array}{l}\text { compression of d. } \\
\text { hepaticus communis, } \\
\text { peritonitis, cholangiogen } \\
\text { sepsis }\end{array}$ & $\begin{array}{l}\text { compression of } d \text {. hepaticus } \\
\text { communis, abscessus } \\
\text { hepatis, liver insufficiency, } \\
\text { septic shock }\end{array}$ \\
\hline outcome & stabilization & no recurrence & $\begin{array}{l}\text { progression, AE related } \\
\text { death }\end{array}$ & $\begin{array}{l}\text { progression, AE related } \\
\text { death }\end{array}$ \\
\hline
\end{tabular}

E.m Echinococcus multilocularis, AE alveolar echinococcosis, CE cystic echinococcosis, $v$ vena, $d$ ductus, AST aspartate aminotransferase, ALT alanine aminotransferase, GGT gamma-glutamyltransferase, ALP alkaline phosphatase, sebi serum bilirubin, US ultrasound, CT computer tomography, MRI magnetic resonance imaging, $I H$ immunohistochemistry using monoclonal antibody $m A b E m 2 G 11, P C R$ polymerase chain reaction, $t x$ treatment, $E P I$ endoscopic and percutaneous interventions, ERCP endoscopic retrograde cholangiopancreatography, PTC percutaneous transhepatic cholangiography, PTD percutaneous transhepatic drainage, FNAB fine needle aspiration biopsy, $S$ liver segment, $A B Z$ albendazole, $H C C$ hepatocellular carcinoma

enzymes (ALP and GGT). Imaging studies (US, CT, MRI) revealed typical hepatic AE lesions in 14 patients (87.5\%). In one probable patient, two interconnected $\mathrm{AE}$ pseudocystic lesions were detected in both lobes with $130 \mathrm{~mm}$ and $120 \mathrm{~mm}$ in diameter. In one confirmed patient, multiple small calcified lesions were identified [22]. Hepatic localization of the parasite and environmental parasitic infiltration at the time of diagnosis was observed in 15 patients $(93.8 \%)$. The disease stage was $\mathrm{P} 1$ in four (25\%), P2 in three (18.8\%), P3 in two (12.5\%) and P4 in six patients $(37.5 \%)$. Extrahepatic involvement of neighboring organs (N1) was detected in three patients (18.8\%). Subphrenic abscess $(n=1 ; 6.3 \%)$, dissemination along falciform ligament $(\mathrm{n}=1 ; 6.3 \%)$ and dissemination along omental peritoneum $(n=1 ; 6.3 \%)$ were present. The absence or presence of distant metastasis was completely evaluated only in six patients (37.5\%). Distant metastasis was not found either of these patients M0 $(n=6 ; 37.5 \%)$. Pulmonary metastasis at the time of diagnosis was excluded in 15 patients (93.8\%). Based on radiological findings, the following preliminary diagnoses were made: echinococcosis, cystic echinococcosis, liver metastasis, sarcoidosis, granulomatous hepatitis, haemangioma, hepatocellular adenoma, hepatocellular carcinoma, intrahepatic cholangiocarcinoma, cystic neoplasm of the liver and fibrolamellar carcinoma. Based on histopathological findings, the following preliminary diagnoses were made: granulomatous hepatitis, chronic hepatitis with fibrosis, helminthosis and echinococcosis. In 10 patients confirmed by histology, protoscoleces or hooklets were evident in two patients (20\%) (Fig. 2c-d). In four patients (25\%), immunohistochemistry $(n=2 ; 12.5 \%)$ and polymerase chain reaction $(\mathrm{n}=2 ; 12.5 \%)$ were used to confirm diagnosis. Em antibodies were detected in 13 out of 16 patients (81.3\%). Diagnostic delay ranged from 1 to 122 months (mean diagnostic delay: 33 months) (Table 1).

\section{Therapeutic features}

Thirteen out of $16 \mathrm{AE}$ patients (81.3\%) received ABZ. Three patients $(18.8 \%)$ received no $A B Z$ treatment due to misdiagnosis (Table 2). During the whole study period, five $\mathrm{AE}$ patients (31.25\%) received a daily dose of $800 \mathrm{mg}$ uninterrupted ABZ treatment from the time of diagnosis because of multiple and/or extended unresectable $\mathrm{AE}$ lesion(s). In one patient (6.3\%), AE was recognized in an advanced stage (P4N1Mx), and the patient died after 3 months of treatment. One unresectable patient (6.3\%) was treated with ABZ for a total of 12 months with long interruptions during his 124 months follow-up period. Three patients $(18.8 \%)$ with unresectable AE lesion(s) received ABZ treatment for less than 4 months from the time of diagnosis. Causes of ceasing 
therapy were drug-related hepatotoxicity, allergic reactions, virtual stabilization of AE lesions and propagation of liver lesions (with supposed uneffectivity of $\mathrm{ABZ}$ treatment). Four patients (25\%) received adjuvant $A B Z$ therapy following removal of $\mathrm{AE}$ lesion(s) by radical (R0) liver surgery. Four patients (25\%) received an incomplete concomitant $\mathrm{ABZ}$ treatment with a total of 5 months duration (Table 2).

Surgery was performed in nine out of $16 \mathrm{AE}$ patients (56.3\%). The timely diagnosis of $\mathrm{AE}$, as a major impact on choosing the proper method of surgical intervention, was only confirmed in one out of the nine patients (6.3\%). In three patients $(18.8 \%)$, explorative laparotomy was carried out for diagnostic purposes to assess resectability and gain tissue-sample for histopathological analysis. Unresectability was detected in three patients $(18.8 \%)$ due to extrahepatic peritoneal dissemination $(n=1 ; 6.3 \%)$, central localization compressing ductus hepaticus communis $(\mathrm{n}=1 ; 6.3 \%)$ and peritoneal dissemination and also the compression of ductus hepaticus communis $(\mathrm{n}=1 ; 6.3 \%)$. Radical resection aiming to excise the entire parasitic lesion with safety margin (R0) were done in five patients (31.3\%) as follows: extended right hemihepatectomy with feeding catheter jejunostomy $(n=1$; $6.3 \%)$, right hemihepatectomy with hepaticojejunostomy $(n=2 ; 12.5 \%)$ and segmentectomy $(n=2 ; 12.5 \%)$. In two patients $(12.5 \%)$ fenestration and marsupialisation of $\mathrm{AE}$ lesions, as palliative methods were performed.

Endoscopic-retrograde-cholangio-pancreatography was performed in five patients (31.3\%) because of $\mathrm{AE}$ associated biliary obstruction. Endoscopic biliary stents were placed five times in three patients. In one patient, nasobiliary stent placement was also necessary to facilitate bile passage. A total of four percutaneous transhepatic drainage of $\mathrm{AE}$ lesions in two patients were performed. Among the five patients $(31.3 \%)$ who needed endoscopic and/or percutaneous interventions, the following stages were determined at diagnosis: P4N1Mx $(\mathrm{n}=2 ; 12.5 \%), \mathrm{P} 4 \mathrm{~N} 0 \mathrm{Mx}$ $(\mathrm{n}=2 ; 12.5 \%)$; P3NOMx $(\mathrm{n}=1 ; 6.3 \%)$ (Table 1; Table 2).

\section{Outcome}

Clinical follow up period ranged from 1 to 177 months (mean 52.4 months). Because of misdiagnosis, the probable first documented Hungarian AE patient was left untreated. Based on the laboratory findings, $\mathrm{AE}$ presumably progressed but lack of pathological investigations did not allow to draw any conclusion on this very first patient. In four patients (25\%), no recurrence of $\mathrm{AE}$ was detected after radical surgery and concomitant $A B Z$ treatment. In these patients, disease free period from curative surgery to the date of final imaging ranged from 10 to 74 months. Stabilization of $\mathrm{AE}$ lesions with continuous ABZ treatment was achieved in three $(60 \%)$ out of five patients with unresectable lesions. One probable $\mathrm{AE}$ patient with multiple hepatic $\mathrm{AE}$ lesions received $A B Z$ treatment for only 3 months. Ten years later, no progression was detected despite the lack of continuous $\mathrm{ABZ}$ therapy. In one probable and unresectable patient intermittent low dose $(2 \times 100 \mathrm{mg}$ per day $)$ of ABZ therapy had to be ceased because of drug-related hepatotoxicity and allergic reactions. After 26 months without treatment, hepatic AE lesions stabilized, but pulmonary microlesions emerged. Unfortunately, the patient was permanently supported with hydrocortisone for hypadrenia, which may have influenced the course of AE. Progression of AE during the study period was proved in seven patients (43.8\%). In our series, progression was not generally accompanied with PNM upstaging of cases but increasing size of $\mathrm{AE}$ lesion(s) and/or worsening clinical condition directly related to AE. In two (33.3\%) out of six patients, short-term or interrupted ABZ treatment is a plausible explanation for progression. In one case, the size of $\mathrm{AE}$ lesion increased beside adequate continuous ABZ treatment. In this patient, disseminating malignant neuroendocrine tumor with liver metastases was diagnosed and simultaneously treated with a probable liver AE lesion. Malignancy, administration of somatostatin analog sandostatin, targeted radionuclide therapy and classical radiotherapy, may play a role in the course of $\mathrm{AE}$ as immunocompromising factors. We registered three AE related deaths (18.8\%) in our study. In patient No. 5 (P4NOMx), giant pseudocystic AE was presumably misdiagnosed as abscessing CE. Endoscopic Retrograde Cholangiopancreatography (ERCP), stent implantation, surgical marsupialisation and drainage were performed. Central biliary obstruction, bilioperitoneal fistula, injury of bile ducts with subsequent bile leaking, complete lack of ABZ treatment, cachexia and advanced age were possible factors contributing to death. In patient No. 15 (P4N1Mx), imaging studies and explorative laparotomy revealed a central unresectable AE lesion $(120 \mathrm{~mm})$ occupying the left lobe and compressing the common hepatic duct with ascites and parasitic invasion along the falciform ligament. $\mathrm{AE}$ was confirmed by histopathology from the surgical sample. Peritonitis and cholangiogen sepsis were the causes of death in this advanced case. In patient No. 16 (P4NOMx), imaging studies revealed a $120 \mathrm{~mm} \mathrm{AE}$ lesion misdiagnosed as cystic echinococcosis, abscess or tumor of the liver occupying the right lobe and compressing the common hepatic duct. ERCP, biliary stent implantation, repeated percutaneous transhepatic drainage and finally right hemihepatectomy were performed with hepaticojejunostomy. Postoperative bleeding, liver failure and septic shock led to the death of the patient. AE was confirmed by histopathology postoperatively from surgical sample. Mean diagnostic delay in the three lethal AE patients was around 10 years (116 months) (Table 1; Table 2).

\section{Discussion}

By the end of the 1980s, the "historic endemic area" where Em was known to occur in foxes was composed 
by four countries (Austria, France, Germany and Switzerland) from Central and Western Europe [24, 25]. In these countries nowadays, the majority of $\mathrm{AE}$ patients have a nearly-normal life expectancy and a good quality of life while treated according to WHO-IWGE guidelines [26-28]. The subsequent increased emergence of Em in European red foxes has been traced back to the increase in fox population size due to antirabies vaccination, change of human attitudes towards foxes and other ecological factors [25]. Examination of foxes performed since 1989 revealed that the European endemic area of Em is much larger than previously assumed, and cases of human AE have been described in countries previously not recognized as endemic. Out of the European "historic endemic area", confirmed and presumably autochthonous human AE cases were reported in Belarus, Belgium, Croatia, Czech Republic, Lithuania, Latvia, Poland, Romania, Slovenia, Slovakia, The Netherlands and Hungary [29-31]. Regarding countries adjacent to Hungary, the first human AE cases were detected in Romania in 1999 [32, 33], Slovakia in 2004 [34], Slovenia between 2001 and 2005 [35] and Croatia in 2014 [36].

In Hungary, Em was first recorded in red foxes near to the Hungarian-Slovak border in the Northern Mountain Range in 2002 [37]. After the first confirmation of this parasite in Hungary, further studies were carried out to determine the burden of this parasitic infection in the major wild definitive host, the red fox. Between 2008 and 2019, Em was detected in 18 out of the 19 Hungarian counties, comprising Budapest, with an average prevalence of $7.6 \%$ in 3265 analyzed red foxes ([38, 39], unpublished). Highest prevalence was detected in the North-Western half of the country (Fig. 3) [38, 39]. As reported in Fig. 3, the spatial distribution of Em in foxes was highly clustered. This spatial distribution pattern of the parasite can be explained by environmental factors. In fact, the mean annual temperature and the annual precipitations resulted as major statistically significant determinants for the spatial distribution of Em in foxes in Hungary [39]. These results can be attributed to the sensitivity of Em eggs to high temperature and desiccation [40]. Similar relationships with temperature or precipitation and Em infection of foxes or water voles were also observed in France, Germany and Switzerland [41-44]. As a consequence of the fox population increase and change of human behavior, red foxes inhabited urban and suburban areas in Hungary [45] as in other Central European countries [46], increasing the risk of human AE infections. Moreover, golden jackals (Canis aureus) have also been identified as definitive hosts in Hungary [47, 48]. Data regarding occurrence of the parasite in intermediate hosts in Hungary are scarce [31]. During a veterinary surveillance study on echinococcosis in livestock conducted between 2015 and 2018, three swine were found to be infected with $\mathrm{Em}$ in Hungary, suggesting that swine cases may be regarded as indicators of the environmental contamination by Em eggs [49].

Regarding human echinococcosis in Hungary, cystic echinococcosis $(\mathrm{CE})$ caused by E. granulosus sensu lato is a well-known parasitic disease since the nineteenth

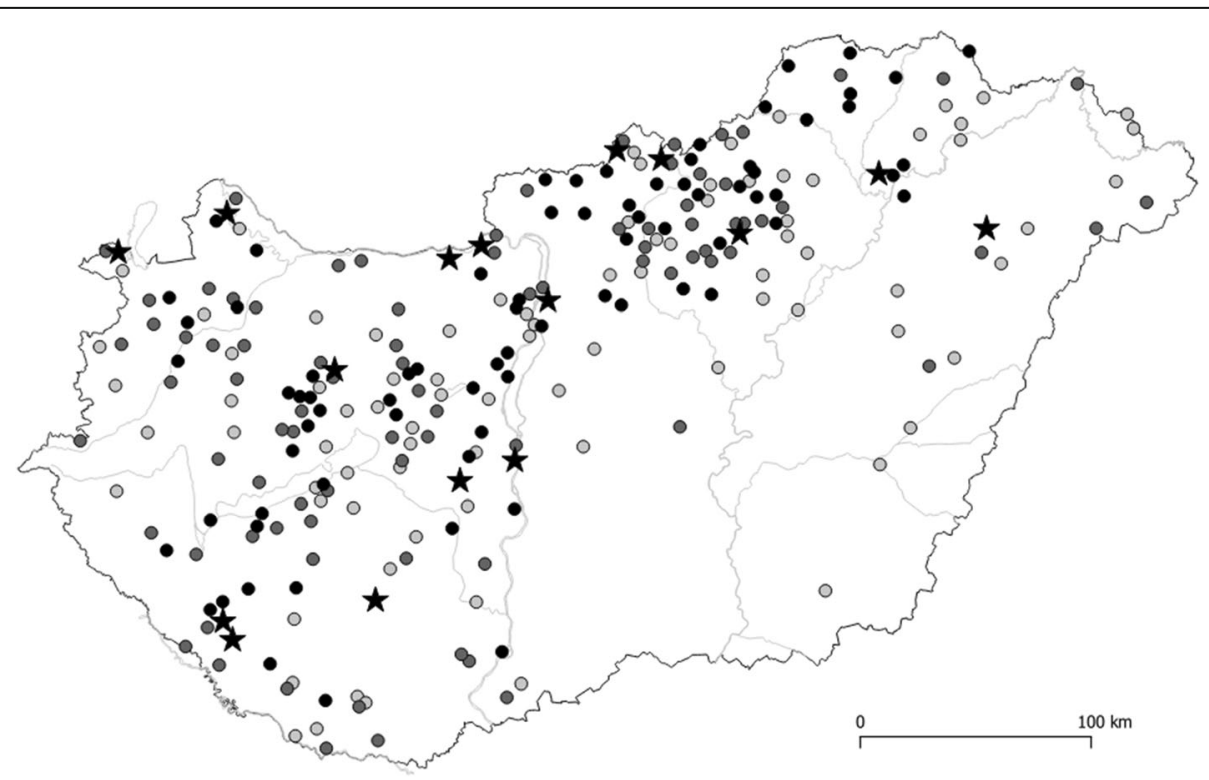

Fig. 3 Spatial distribution of 16 AE human cases (stars) and that of 247 red foxes (Vulpes vulpes) (dots) infected with Echinococcus multilocularis out of 3265 foxes examined between 2008 and 2019. The darkness of the dots reflects the intensity of infection in foxes (light grey: < 10 worms; grey: 1-100 worms; black: > 100 worms) Aapproximately 4\% of the fox population of each county was sampled 
century. In fact, $\mathrm{CE}$ is the most prevalent reportable zoonotic helminthosis in Hungary, which is endemic in several regions with considerable disease burden [50]. In contrast, despite the prevalence of Em in foxes, the first confirmed human AE patient in Hungary was reported only in 2008 [51], and the first confirmed autochthonous human AE case was described in 2016 [22]. Herein we reported further $14 \mathrm{AE}$ cases. The limitation of our study may rely on sampling bias, possibly underestimating $\mathrm{AE}$ cases. In fact, vast majority of our case series (94\%) were collected from the official registry of $\mathrm{Na}$ tional Public Health Centre, which is only based on seropositivity for Em. Inadequacies in the reporting system [50] have resulted in the lack of reporting of seronegative AE cases, which might had been diagnosed exclusively by histopathology. Therefore, we cannot exclude that the true number of $\mathrm{AE}$ cases might have been higher in Hungary in the past decade. A matter of debate whether the emergence of human $\mathrm{AE}$ in Europe is a consequence of the expansion of the classical endemic area through increasing number of fox populations and consequently infected animals or the result of improved awareness and better availability of diagnostic tools. In Hungary, the spread and emergence of Em could be observed in both animals and humans in the past two decades [37-39, 47-49]. Our results showed that the residence of human $\mathrm{AE}$ patients overlaps the geographical distribution of Em infected red foxes in Hungary (Fig. 3), suggesting that the majority of human cases may be autochthonous. The majority of $\mathrm{AE}$ patients $(n=11 ; 68,8 \%)$ originated from rural or suburban areas, which is in line with the observations by other researchers [46] and supporting the hypothesis that living in rural areas may be a proxy for environmental contamination and a driver for AE infection [4]. Regarding other main potential risk factors, almost three quarters of the patients had kitchen garden or visited forests for vocational reasons.

The incidence of human AE is low in Hungary and it is often misdiagnosed as $\mathrm{CE}$, liver abscess or malignancy of the hepatobiliary tract, and most of the patients are only recognized in an advanced and unresectable stage. Six patients (37.5\%) were diagnosed with hilar extension of the parasite (P4) and lethality reached $50 \%$ in this subgroup. PNM system is useful for AE treatment according WHOIWGE and assessing prognosis. Retrospective application of PNM has made our results partially comparable on an international level [19]. Unfortunately, ${ }^{18}$ F-FDG-PET/CT, which has found to be useful in the follow-up of AE [5254 ], is currently not covered by the health insurance system for patients with non-malignant conditions in Hungary. Furthermore, the lack of complete and detailed $\mathrm{CT}$ imaging data did not allow an advanced typing of $\mathrm{AE}$ lesions, like EMUC-CT classification [13, 55].
Diagnosis of $\mathrm{AE}$ was often delayed (mean diagnostic delay was 33 months in this series) because of misdiagnosis. Hepatobiliary malignancies, especially intrahepatic cholangiocarcinoma (ICC) were frequent misdiagnoses in our AE series. Enhancement pattern and matrix calcifications are the only imaging criteria with a high discriminating power between AE and ICC. During imaging evaluation, the combined presence of no or septal enhancement and calcification ensures $100 \%$ specificity for AE [56]. In our series, no or septal enhancement and/or calcification were detectable during preliminary imaging evaluation (CT and MRI) in 11 out of 16 patients (68.8\%), which would have allowed the radiologists to distinguish AE from ICC. Non-malignancies such as $\mathrm{CE}$ are also pitfalls during differential diagnosis, not allowing further investigations and contributing to diagnostic delay [57]. We emphasize that the awareness of specific imaging characteristics of $\mathrm{AE}$ could help to reduce diagnostic delay and accelerate the introduction of antiparasitic drug treatment. In most patients, diagnostic uncertainty or missing histological confirmation led to short-term/interrupted or complete lack of $\mathrm{ABZ}$ treatment and to inappropriate surgical interventions, including radical liver surgery with severe complications, which is in line with previous observations made by other researchers [57]. Endoscopic and percutaneous interventions were generally used in defined advanced $\mathrm{AE}$ patients. We registered three lethal cases of human $\mathrm{AE}$ in our study (fatality rate 18.8\%). Since 2005, only three lethal cases caused by autochthonous parasitic infections have been recognized in Hungary, all of them were involved in this series. Misdiagnosis or diagnostic delay may have brought to an inadequate treatment of these three $\mathrm{AE}$ cases, resulting in an increased death rate due to palliative surgery or lack of proper medical treatment $[58,59]$. In fact, before the advent of medical treatment with benzimidazoles, fatality rate exceeded $90 \%$ of $\mathrm{AE}$ cases within $10-15$ years from diagnosis [60]. The major current causes of death due to $\mathrm{AE}$ are either septic shock, liver failure, complications after major liver surgery and complications due to secondary biliary cirrhosis $[9,61-63]$.

\section{Conclusions}

We conclude that $\mathrm{AE}$ is currently the most dangerous human parasitic infection in Hungary. Our results highlight the need of early differential diagnosis supported by accurate imaging evaluation and if serology is not conclusive, additional core-biopsies from progressive liver lesions for histopathological analysis are needed. Histology is a corner stone to distinguish AE from malignancies, bacterial liver abscess and $\mathrm{CE}$, which is also endemic and much more prevalent in Hungary than AE [64]. Early diagnosis and staging of $\mathrm{AE}$ allow applying evidence-based treatment methods according to WHO-IWGE and may increase 
cure rates [28]. ABZ lifelong treatment prevents disease progression and leads to a favorable outcome in most patients. Stage-specific treatment avoids inadequate/palliativeinterventions and potential complications associated with these procedures [61]. The course of $\mathrm{AE}$ in immunosuppressed patients needs further observations and precise evaluation [65]. As the results of this study indicate, there is an urgent need for the education of physicians in the diagnosis and clinical management of $\mathrm{AE}$ in Hungary and in other newly endemic European countries. This is very important since in historic endemic European countries it has been shown a favorable outcome of AE-patients with adequate treatment.

\section{Acknowledgements}

We are grateful to István Kucsera (Head of the National Reference Laboratory for Human Parasitic Diseases, National Public Health Centre, Budapest, Hungary) for supporting this research. We are also thankful to György Somorácz (Saint Pantaleon Teaching Hospital, Dunaújváros, Hungary), Andrea Horváth, Szilvia Tóth and Katalin Fried (Central Hospital of Southern Pest National Institute of Hematology and Infectious Diseases, Budapest, Hungary), Balázs Nemes and Attila Doros (Department of Transplantation and Surgery, Semmelweis University, Budapest, Hungary), Tünde Nagy and Tamás Mersich (National Institute of Oncology, Budapest, Hungary), László Nehéz (1st Department of Surgery, Semmelweis University, Budapest, Hungary), Mónika Francz (Jósa András County Teaching Hospital, Nyíregyháza, Hungary), Judit Kovács (Borsod-Abaúj-Zemplén County Teaching Hospital, Miskolc, Hungary), Prof. Ákos Pap and Prof. Béla Hunyady (Somogy County Teaching Hospital, Kaposvár, Hungary), Albert Szabó (Petz Aladár County Teaching Hospital, Győr, Hungary) for their contribution to the diagnosis and treatment of AE patients involved in this study. We also thank the colleagues who volunteered to help with retrieving official human data from Hungary: Dóra Somorácz-Kiss, Levente Kővágó, Tibor Martyin and Piroska Lakatos.

\section{Authors' contributions}

BD conceived and designed the study. BD, ZK, TS, BG, ACa and TFEB drafted the work. BD, ZD, TS, EC, VC, ZK, MF, AS, ACS, AO, KA, AP, DG, ZS, ZT, TS, JD and $H A$ acquired the data. $B D, Z D, T S, E C, V C, Z K, M F, A S, A C s, A O, K A, A P$, $D G, Z S, Z T, T S, J D, H A, B G$, TFEB and ACa critically revised the work. All authors have approved the submitted version. All authors have agreed to be personally accountable for the author's own contributions and to ensure that questions related to the accuracy or integrity of any part of the work, even ones in which the author was not personally involved, are appropriately investigated, resolved, and the resolution documented in the literature.

\section{Funding}

The research that will lead to these results was partially supported by ERANet-LAC 2nd Joint Call (http://www.eranet-lac.eu) and the Italian Ministry of Health - NDTND project. The funding sources had no involvement in the preparation, ideas, writing, interpretation, or the decision to submit this article.

\section{Availability of data and materials}

The data that support the findings of this study are available at the database management systems of participating study centres but restrictions apply to the availability of these data, which were used under license for the current study and so are not publicly available. Data are however available from the authors upon reasonable request and with permission of the participating institutional ethical boards.

\section{Ethics approval}

This study was approved by all of the participating institutional ethical boards and by the Scientific and Research Ethics Committee of the Hungarian Medical Research Council (48967-5/2019/EKU). Data regarding potential risk factors were collected by means of questionnaires with dichotomous questions delivered to patients providing informed consent.

\section{Consent for publication}

Not applicable.

\section{Competing interests}

The authors declared no competing interests.

\section{Author details}

${ }^{1}$ Temporarily unaffiliated, Budapest, Hungary. ${ }^{2}$ National Institute of Oncology, Tumour Surgery Centre, Budapest, Hungary. ${ }^{3}$ National Institute of Oncology, Centre of Surgical and Molecular Tumor Pathology, Budapest, Hungary. ${ }^{4}$ Somogy County Teaching Hospital, Department of Surgery, Kaposvár, Hungary. ${ }^{5}$ Somogy County Teaching Hospital, Department of Pathology, Kaposvár, Hungary. ${ }^{6}$ Jósa András County Teaching Hospital, 4th Department of Internal Medicine, Nyíregyháza, Hungary. ${ }^{7}$ 2nd Department of Pathology, Semmelweis University, Budapest, Hungary. ${ }^{8}$ Borsod-Abaúj-Zemplén County Teaching Hospital, Department of Infectology, Miskolc, Hungary. ${ }^{9}$ Petz Aladár County Teaching Hospital, Department of Surgery, Győr, Hungary. ${ }^{10} \mathrm{Petz}$ Aladár County Teaching Hospital, Department of Pathology, Györ, Hungary.

${ }^{11}$ Department of Transplantation and Surgery, Semmelweis University, Budapest, Hungary. ${ }^{12}$ National Reference Laboratory for Parasites, Fish and Bee Diseases, National Food Chain Safety Office, Budapest, Hungary.

${ }^{13}$ National Reference Laboratory for Human Parasitic Diseases, National Public Health Centre, Budapest, Hungary. ${ }^{14}$ Department of Medical Parasitology, Centre of Pathophysiology, Infectology and Immunology, Institute of Specific Prophylaxis and Tropical Medicine, Medical University Vienna, Vienna, Austria. ${ }^{15}$ Division of Clinical Infectious Diseases, Department of Internal Medicine III, University Hospital UIm, Ulm, Germany. ${ }^{16}$ Department of Pathology, Ulm University, Ulm, Germany. ${ }^{17}$ WHO Collaborating Centre for the epidemiology, detection and control of cystic and alveolar echinococcosis, Department of Infectious Diseases, Istituto Superiore di Sanità, Rome, Italy. ${ }^{18}$ European Union Reference Laboratory for Parasites. Department of Infectious Diseases, Istituto Superiore di Sanità, Rome, Italy.

Received: 1 October 2020 Accepted: 31 January 2021

Published online: 10 February 2021

\section{References}

1. Kern P, Bardonnet K, Renner E, Auer H, Pawlowski Z, Ammann RW, et al. European echinococcosis registry: human alveolar echinococcosis, Europe, 1982-2000. Emerg Infect Dis. 2003;9:343-9. https://doi.org/10.3201/eid0903.020341.

2. Oksanen A, Siles-Lucas M, Karamon J, Possenti A, Conraths FJ, Romig T, et al. The geographical distribution and prevalence of Echinococcus multilocularis in animals in the European Union and adjacent countries: a systematic review and meta-analysis. Parasit Vectors. 2016;9:519. https://doi.org/10. 1186/s13071-016-1746-4.

3. Casulli A, Barth TFE, Tamarozzi F. Echinococcus multilocularis. Trends Parasitol. 2019;35:738-9. https://doi.org/10.1016/j.pt.2019.05.005.

4. Conraths FJ, Probst C, Possenti A, Boufana B, Saulle R, La Torre G, Busani L, Casulli A. Potential risk factors associated with human alveolar echinococcosis: systematic review and meta-analysis. PLoS Negl Trop Dis. 2017;11(7):e0005801. https://doi.org/10.1371/journal.pntd.0005801.

5. Torgerson PR, Robertson L, Enemark HL, Foehr J, van der Giessen JWB, Kapel CMO, Klun I, Trevisan C. Source attribution of human echinococcosis: a systematic review and meta-analysis. Plos Negl Trop Dis. 2020;14(6): e0008382.

6. Tamarozzi F, Deplazes P, Casulli A. Reinventing the wheel of Echinococcus granulosus sensu lato transmission to humans. Trends Parasitol. 2020;36(5): 427-34. https://doi.org/10.1016/j.pt.2020.02.004.

7. Brunetti E, Kern P, Vuitton DA. Writing panel for the WHO IWGE. Expert consensus for the diagnosis and treatment of cystic and alveolar echinococcosis in humans. Acta Trop. 2009;114:10-6. https://doi.org/10. 1016/j.actatropica.2009.11.001.

8. Kratzer W, Gruener B, Kaltenbach TE, Ansari-Bitzenberger S, Kern P, Fuchs M, Mason RA, Barth TF, Haenle MM, Hillenbrand A, Oeztuerk S, Graeter T. Proposal of an ultrasonographic classification for hepatic alveolar echinococcosis: Echinococcosis multilocularis Ulm classification-ultrasound. World J Gastroenterol. 2015;21(43):12392-402. https://doi.org/10.3748/wjg. v21.143.12392.

9. Bresson-Hadni S, Vuitton DA, Bartholomot B, Heyd B, Godart D, Meyer JP, Hrusovsky S, Becker MC, Mantion G, Lenys D, Miguet JP. A twenty year history of alveolar echinococcosis: analysis of a series of 117 patients from 
eastern France. Eur J Gastroenterol Hepatol. 2000;12:327-36. https://doi.org/ 10.1097/00042737-200012030-00011.

10. Bresson-Hadni S, Delabrousse E, Blagosklonov O, Bartholomot B, Koch S, Miguet JP, Mantion GA, Vuitton DA. Imaging aspects and non-surgical interventional treatment in human alveolar echinococcosis. Parasitol Int. 2006:55(Suppl):S267-72. https://doi.org/10.1016/.parint.2005.11.053.

11. Reuter S, Nüssle K, Kolokythas O, Haug U, Rieber A, Kern P, Kratzer W. Alveolar liver echinococcosis: a comparative study of three imaging techniques. Infection. 2001;29(3):119-25. https://doi.org/10.1007/s15010-001-1081-2.

12. Kodama Y, Fujita N, Shimizu T, Endo H, Nambu T, Sato N, Todo S, Miyasaka K. Alveolar echinococcosis: MR findings in the liver. Radiology. 2003;228(1): 172-7. https://doi.org/10.1148/radiol.2281020323.

13. Graeter T, Kratzer W, Oeztuerk S, Haenle MM, Mason RA, Hillenbrand A, Kull T, Barth TF, Kern P, Gruener B. Proposal of a computed tomography classification for hepatic alveolar echinococcosis. World J Gastroenterol. 2016;22(13):3621-31. https://doi.org/10.3748/wjg.v22.113.3621.

14. Kantarci M, Bayraktutan U, Karabulut N, Aydinli B, Ogul H, Yuce I, Calik M, Eren S, Atamanalp SS, Oto A. Alveolar echinococcosis: spectrum of findings at cross-sectional imaging. Radiographics. 2012;32(7):2053-70. https://doi. org/10.1148/rg.327125708.

15. Bulakçı M, Kartal MG, Yılmaz S, Yılmaz E, Yılmaz R, Şahin D, Aşık M, Erol OB. Multimodality imaging in diagnosis and management of alveolar echinococcosis: an update. Diagn Interv Radiol. 2016;22(3):247-56. https:// doi.org/10.5152/dir.2015.15456.

16. Tamarozzi F, Horton J, Muhtarov M, Ramharter M, Siles-Lucas M, Gruener B, Vuitton DA, Bresson-Hadni S, Manciulli T, Brunetti E. A case for adoption of continuous albendazole treatment regimen for human echinococcal infections. Plos Negl Trop Dis. 2020;14(9):e0008566. https://doi.org/10.1371/ journal.pntd.0008566 PMID: 32941434; PMCID: PMC7498015.

17. Siles-Lucas M, Casulli A, Cirilli R, Carmena D. Progress in the pharmacological treatment of human cystic and alveolar echinococcosis: Compounds and therapeutic targets. Plos Negl Trop Dis. 2018;12(4):e0006422. https://doi.org/ 10.1371/journal.pntd.0006422 PMID: 29677189; PMCID: PMC5931691.

18. Kern P. Clinical features and treatment of alveolar echinococcosis. Curr Opin Infect Dis. 2010;23(5):505-12. https://doi.org/10.1097/QCO. 0b013e32833d7516.

19. Kern $\mathrm{P}$, Wen $\mathrm{H}$, Sato $\mathrm{N}$, Vuitton DA, Gruener B, Shao Y, et al. WHO classification of alveolar echinococcosis: principles and application. Parasitol Int. 2006;55(S):283-7. https://doi.org/10.1016/.jparint.2005.11.041.

20. Koch S, Bresson-Hadni S, Miguet JP, Crumbach JP, Gillet M, Mantion GA, Heyd B, Vuitton DA, Minello A, Kurtz S. European collaborating clinicians. Experience of liver transplantation for incurable alveolar echinococcosis: a 45-case European collaborative report. Transplantation. 2003;75(6):856-63. https://doi.org/10.1097/01.TP.0000054230.63568.79.

21. Liance M, Janin V, Bresson-Hadni S, Vuitton DA, Houin R, Piarroux R. Immunodiagnosis of Echinococcus infections: confirmatory testing and species differentiation by a new commercial Western blot. J Clin Microbiol. 2000;38(10):3718-21. https://doi.org/10.1128/JCM.38.10.3718-3721.2000.

22. Dezsényi B, Strausz T, Makrai Z, Csomor J, Danka J, Kern P, et al. Autochthonous human alveolar echinococcosis in a Hungarian patient. Infection. 2017:45:107-10. https://doi.org/10.1007/s15010-016-0918-7.

23. Barth TFE, Herrmann TS, Tappe D, Stark L, Grüner B, Buttenschoen K. Sensitive and specific immunohistochemical diagnosis of human alveolar echinococcosis with the monoclonal antibody Em2G11. Plos Negl Trop Dis. 2012;6:e1877. https://doi.org/10.1371/journal.pntd.0001877.

24. Eckert J, Deplazes P. Alveolar echinococcosis in humans: the current situation in Central Europe and the need for countermeasures. Parasitol Today. 1999;15(8):315-9. https://doi.org/10.1016/s0169-4758(99)01476-3.

25. Schweiger A, Ammann RW, Candinas D, Clavien PA, Eckert J, Gottstein B, et al. Human alveolar echinococcosis after fox population increase, Switzerland. Emerg Infect Dis. 2007;13:878-82. https://doi.org/10.3201/eid1306.061074.

26. Torgerson PR, Schweiger A, Deplazes P, Pohar M, Reichen J, Ammann RW, Tarr PE, Halkic N, Müllhaupt B. Alveolar echinococcosis: from a deadly disease to a well-controlled infection. Relative survival and economic analysis in Switzerland over the last 35 years. J Hepatol. 2008;49(1):72-7. https://doi.org/10.1016/j.jhep.2008.03.023.

27. Piarroux M, Piarroux R, Giorgi R, Knapp J, Bardonnet K, Sudre B, Watelet J, Dumortier J, Gérard A, Beytout J, Abergel A, Mantion G, Vuitton DA, Bresson-Hadni S. Clinical features and evolution of alveolar echinococcosis in France from 1982 to 2007: results of a survey in 387 patients. J Hepatol. 2011;55(5):1025-33. https://doi.org/10.1016/j.jhep.2011.02.018.
28. Grüner B, Kern P, Mayer B, Gräter T, Hillenbrand A, TEF B, Muche R, HenneBruns D, Kratzer W, Kern P. Comprehensive diagnosis and treatment of alveolar echinococcosis: A single-center, long-term observational study of 312 patients in Germany. GMS Infect Dis. 2017;5:Doc01. https://doi.org/10.3205/id000027.

29. Baumann S, Shi R, Liu W, Bao H, Schmidberger J, Kratzer W, Li W, the Interdisciplinary Echinococcosis Working Group Ulm. Worldwide literature on epidemiology of human alveolar echinococcosis: a systematic review of research published in the twenty-first century. Infection. 2019;47:703-27. https://doi.org/10.1007/s15010-019-01325-2.

30. Vuitton DA, Demonmerot F, Knapp J, Richou C, Grenouillet F, Chauchet A, Vuitton L, Bresson-Hadni S, Millon L. Clinical epidemiology of human AE in Europe. Vet Parasitol. 2015;213(3-4):110-20. https://doi.org/10.1016/j.vetpar. 2015.07.036.

31. Deplazes P, Rinaldi L, Alvarez Rojas CA, Torgerson PR, Harandi MF, Romig T, Antolova D, Schurer JM, Lahmar S, Cringoli G, Magambo J, Thompson RC, Jenkins EJ. Global distribution of alveolar and cystic Echinococcosis. Adv Parasitol. 2017:95:315-493. https://doi.org/10.1016/bs.apar.2016.11.001.

32. Panaitescu D, Pop M. Alveococoza la om. [Alveococcosis in man]. Rev Rom Parazitol. 1999;9(2):55 [In Romanian].

33. Sikó SB, Deplazes P, Ceica C, Tivadar CS, Bogolin I, Popescu S, Cozma V. Echinococcus multilocularis in South-Eastern Europe (Romania). Parasitol Res. 2011;108(5):1093-7. https://doi.org/10.1007/s00436-010-2150-1.

34. Antolova D, Miterpakova M, Radoňak J, Hudačkova D, Szilagyiova M, Začek M. Alveolar echinococcosis in a highly endemic area of northern Slovakia between 2000 and 2013. Euro Surveill. 2014;19(34):20882. https://doi.org/10. 2807/1560-7917.ES2014.19.34.20882

35. Logar J, Soba B, Lejko-Zupanc T, Kotar T. Human alveolar echinococcosis in Slovenia. Clin Microbiol Infect. 2007;13(5):544-6. https://doi.org/10.1111/j. 1469-0691.2007.01701.x.

36. Dušek D, Vince A, Kurelac I, Papić N, Višković K, Deplazes P, Beck R. Human Alveolar Echinococcosis. Croatia Emerg Infect Dis. 2020;26(2):364-6. https:// doi.org/10.3201/eid2602.181826.

37. Sréter T, Széll Z, Zs E, Varga I. Echinococcus multilocularis: an emerging pathogen in Hungary and Central Eastern Europe? Emerg Infect Dis. 2003;9: 384-6. https://doi.org/10.3201/eid0903.020320.

38. Casulli A, Széll Z, Pozio E, Sréter T. Spatial distribution and genetic diversity of Echinococcus multilocularis in Hungary. Vet Parasitol. 2010;174:241-6. https://doi.org/10.1016/j.vetpar.2010.08.023.

39. Tolnai Z, Széll Z, Sréter T. Environmental determinants of the spatial distribution of Echinococcus multilocularis in Hungary. Vet Parasitol. 2013;198: 292-7. https://doi.org/10.1016/j.vetpar.2013.09.004.

40. Viel JF, Giraudoux P, Abrial V, Bresson-Hadni S. Water vole (Arvicola terrestris scherman) density as risk factor for human alveolar echinococcosis. Am J Trop Med Hyg. 1999;61 (4):559-65. https://doi.org/10.4269/ajtmh.1999.61.559.

41. Denzin N, Schliephake A, Fröhlich A, Ziller M, Conraths FJ. On the move? Echinococcus multilocularis in red foxes of Saxony-Anhalt (Germany). Transbound Emerg Dis. 2014;61(3):239-46. https://doi.org/10. 1111/tbed.12026.

42. Fischer I, Graeter T, Kratzer W, Stark K, Schlingeloff P, Schmidberger J. Echinococcosis working group Ulm. Distribution of alveolar echinococcosis according to environmental and geographical factors in Germany, 19922018. Acta Trop. 2020;212:105654. https://doi.org/10.1016/j.actatropica.2020. 105654.

43. Chauchet A, Grenouillet F, Knapp J, Richou C, Delabrousse E, Dentan C, Millon L, Di Martino V, Contreras R, Deconinck E, Blagosklonov O, Vuitton DA, Bresson-Hadni S. FrancEchino network. Increased incidence and characteristics of alveolar echinococcosis in patients with immunosuppression-associated conditions. Clin Infect Dis. 2014;59(8):1095-104. https://doi.org/10.1093/cid/ciu520.

44. Burlet $P$, Deplazes $P$, Hegglin D. Age, season and spatio-temporal factors affecting the prevalence of Echinococcus multilocularis and Taenia taeniaeformis in Anvicola terrestris. Parasit Vectors. 2011;:46. https://doi.org/10.1186/1756-3305-4-6.

45. Szemethy L, Heltai M, Zs B. Effect of oral immunization against rabies on the red fox population in Hungary. Rabies Bull Eur. 1999;23:12.

46. Deplazes $\mathrm{P}$, Hegglin D, Gloor S, Romig T. Wilderness in the city: urbanization of Echinococcus multilocularis. Trends Parasitol. 2004;20:77-84. https://doi. org/10.1016/j.pt.2003.11.011.

47. Széll Z, Marucci G, Pozio E, Sréter T. Echinococcus multilocularis and Trichinella spiralis in golden jackals (Canis aureus) of Hungary. Vet Parasitol. 2013;197:393-6. https://doi.org/10.1016/j.vetpar.2013.04.032.

48. Balog T, Nagy G, Halász T, Csányi E, Zomborszky Z, Csivincsik Á. The occurrence of Echinococcus spp. in golden jackal (Canis aureus) in 
southwestern Hungary: Should we need to rethink its expansion? Parasito Int. 2020;80:102214. https://doi.org/10.1016/.jparint.2020.102214.

49. Dán Á, Rónai Z, Széll Z, Sréter T. Prevalence and genetic characterization of Echinococcus spp. in cattle, sheep and swine in Hungary. Parasitol Res. 2018; 117:3019-22. https://doi.org/10.1007/s00436-018-5977-5.

50. Dezsényi B, Somorácz Á, Danka J, Kucsera I, Barth TFE, Casulli A. Human cystic echinococcosis in Hungary (2000-2014): a retrospective case series analysis from a single-center study. Infection. 2018;46:477-86. https://doi. org/10.1007/s15010-018-1146-0.

51. Horváth A, Patonai A, Bánhegyi D, Szlávik J, Balázs GY, Görög D, et al. A humán Echinococcus multilocularis infectio első hazai esete. Orv Hetil. 2008; 149:795-9 [In Hungarian].

52. Reuter S, Schirrmeister H, Kratzer W, Dreweck C, Reske SN, Kern P. Pericystic metabolic activity in alveolar echinococcosis: assessment and follow-up by positron emission tomography. Clin Infect Dis. 1999;29(5):1157-63. https:// doi.org/10.1086/313438.

53. Reuter S, Buck A, Manfras B, Kratzer W, Seitz HM, Darge K, Reske SN, Kern P. Structured treatment interruption in patients with alveolar echinococcosis. Hepatology. 2004;39(2):509-17. https://doi.org/10.1002/hep.20078.

54. Ammann RW, Stumpe KD, Grimm F, Deplazes P, Huber S, Bertogg K, Fischer DR, Müllhaupt B. Outcome after discontinuing long-term Benzimidazole treatment in 11 patients with non-resectable alveolar Echinococcosis with negative FDG-PET/CT and anti-Emll/3-10 serology. Plos Negl Trop Dis. 2015; 9(9):e0003964. https://doi.org/10.1371/journal.pntd.0003964

55. Graeter T, Bao H, Delabrousse E, Brumpt E, Shi R, Li W, Jiang Y, Schmidberger J, Kratzer W, Liu W, XUUB consortium. Hepatic alveolar echinococcosis: Comparative computed tomography study between two Chinese and two European centres. Food Waterborne Parasitol. 2020;19: e00082. https://doi.org/10.1016/j.fawpar.2020.e00082.

56. Mueller J, Stojkovic M, Berger AK, Rosenberger KD, Schlett CL, Kauczor HU, Junghanss T, Weber TF. How to not miss alveolar echinococcosis in hepatic lesions suspicious for cholangiocellular carcinoma. Abdom Radiol (NY). 2016; 41(2):221-30. https://doi.org/10.1007/s00261-015-0561-2.

57. Stojkovic M, Mickan C, Weber TF, Junghanss T. Pitfalls in diagnosis and treatment of alveolar echinococcosis: a sentinel case series. BMJ Open Gastroenterol. 2015;2(1):e000036. https:/doi.org/10.1136/bmjgast-2015-000036.

58. Buttenschoen K, Gruener B, Carli Buttenschoen D, Reuter S, Henne-Bruns D, Kern P. Palliative operation for the treatment of alveolar echinococcosis. Langenbeck's Arch Surg. 2009;394(1):199-204. https://doi.org/10.1007/s00423-008-0367-6.

59. Hillenbrand A, Gruener B, Kratzer W, Kern P, Graeter T, Barth TF, Buttenschoen K, Henne-Bruns D. Impact of safe distance on long-term outcome after surgical therapy of alveolar Echinococcosis. World I Surg. 2017;41(4):1012-8. https://doi.org/10.1007/s00268-016-3813-6.

60. Wilson JF, Rausch RL, McMahon BJ, Schantz PM. Parasiticidal effect of chemotherapy in alveolar hydatid disease: review of experience with mebendazole and albendazole in Alaskan Eskimos. Clin Infect Dis. 1992; 15(2):234-49. https://doi.org/10.1093/clinids/15.2.234.

61. Frei P, Misselwitz B, Prakash MK, Schoepfer AM, Prinz Vavricka BM, Müllhaupt B, Fried M, Lehmann K, Ammann RW, Vavricka SR. Late biliary complications in human alveolar echinococcosis are associated with high mortality. World J Gastroenterol. 2014;20(19):5881-8. https://doi.org/10.3748/wjg.v20.119.5881.

62. Graeter T, Ehing F, Oeztuerk S, Mason RA, Haenle MM, Kratzer W, Seufferlein T, Gruener B. Hepatobiliary complications of alveolar echinococcosis: a longterm follow-up study. World I Gastroenterol. 2015;21(16):4925-32. https:// doi.org/10.3748/wjg.v21.i16.4925.

63. Ambregna S, Koch S, Sulz MC, Grüner B, Öztürk S, Chevaux JB, Sulima M, de Gottardi A, Napoléon B, Abergel A, Bichard P, Boytchev I, Deprez P,

Dumortier J, Frossard JL, Kull E, Meny B, Moradpour D, Prat F, Vanbiervliet G, Thevenot T, Vuitton DA, Bresson-Hadni S, Vuitton L. A European survey of perendoscopic treatment of biliary complications in patients with alveolar echinococcosis. Expert Rev Anti-Infect Ther. 2017;15(1):79-88. https://doi. org/10.1080/14787210.2017.1252260.

64. Reinehr M, Micheloud C, Grimm F, Kronenberg PA, Grimm J, Beck A, Nell J, Meyer Zu Schwabedissen C, Furrer E, Müllhaupt B, Barth TFE, Deplazes P. Weber A. Pathology of Echinococcosis: a morphologic and Immunohistochemical study on 138 specimens with focus on the differential diagnosis between cystic and alveolar Echinococcosis. Am I Surg Pathol. 2020; 44(1):43-54. https://doi.org/10.1097/PAS.0000000000001374 PMID: 31567204.

65. Piarroux M, Gaudart J, Bresson-Hadni S, Bardonnet K, Faucher B, Grenouillet F, Knapp J, Dumortier J, Watelet J, Gerard A, Beytout J, Abergel A, Wallon M, Vuitton DA, Piarroux R. FrancEchino network. Landscape and climatic characteristics associated with human alveolar echinococcosis in France, 1982 to 2007. Euro Surveill. 2015;20(18):21118. https://doi.org/10.2807/15607917.es2015.20.18.21118.

\section{Publisher's Note}

Springer Nature remains neutral with regard to jurisdictional claims in published maps and institutional affiliations.
Ready to submit your research? Choose BMC and benefit from:

- fast, convenient online submission

- thorough peer review by experienced researchers in your field

- rapid publication on acceptance

- support for research data, including large and complex data types

- gold Open Access which fosters wider collaboration and increased citations

- maximum visibility for your research: over $100 \mathrm{M}$ website views per year

At $\mathrm{BMC}$, research is always in progress.

Learn more biomedcentral.com/submissions 